\title{
Can Wernicke's Encephalopathy Be Precipitated by Administering Glucose before Thiamine in Severely Malnourished or Alcoholic Patients?
}

\author{
Joshua Altman, Matthew F. Ryan* \\ Department of Emergency Medicine, University of Florida, Gainesville, FL, USA \\ Email: *mfryan@ufl.edu
}

How to cite this paper: Altman, J. and Ryan, M.F. (2019) Can Wernicke's Encephalopathy Be Precipitated by Administering Glucose before Thiamine in Severely Malnourished or Alcoholic Patients? Case Reports in Clinical Medicine, 8, 245-249. https://doi.org/10.4236/crcm.2019.89030

Received: November 26, 2018

Accepted: September 15, 2019

Published: September 18, 2019

Copyright ( 2019 by author(s) and Scientific Research Publishing Inc. This work is licensed under the Creative Commons Attribution International License (CC BY 4.0).

http://creativecommons.org/licenses/by/4.0/

\begin{abstract}
We discuss the safety and controversy regarding the administration of glucose before thiamine with regards to precipitation of Wernicke's Encephalopathy in the severely malnourished and alcoholic patient population. Herein we review clinical features, pathophysiology and the relevant literature to provide an evidenced-based recommendation that thiamine replacement should not delay glucose administration acutely in the malnourished patient population.
\end{abstract}

\section{Keywords}

Wernicke's Encephalopathy, Thiamine, Glucose

\section{Introduction}

Can Wernicke's Encephalopathy be precipitated by administering glucose before thiamine in severely malnourished or alcoholic patients? The answer is not obvious and the bottom line is that physiologically, this can happen but most patients already have the manifestations of encephalopathy before they present to the emergency department. Moreover, the process does not happen immediately but rather evolves throughout a hospital course as documented in case reports and literature reviews. Current established guidelines are straightforward with the recommendation to not delay glucose administration while waiting for thiamine. In this article we review the current literature and case reports behind the controversy that still exists and make a case for why administration of glucose should not be delayed while waiting for thiamine in the emergency department 
or other acute care settings.

\section{Discussion}

Thiamine deficiency manifests itself as a disease of the central and peripheral nervous system, cardiovascular system, and the gastrointestinal tract. Wernicke's Encephalopathy is a well known, serious complication of thiamine (Vitamin B1) deficiency characterized by a syndrome of altered mental status, ophthalmoplegia, and gait ataxia. Some other thiamine deficiency diseases are listed in Table 1.

The pathophysiology behind the disease relates to thiamine's key role in glucose metabolism. Thiamine is a cofactor for several enzymes in the TCA cycle including $\alpha$-ketoglutarate dehydrogenase, pyruvate dehydrogenase, and transketolase. Thiamine is utilized after phosphorylation to thiamine pyrophosphate. In particular, a decrease in enzymatic activity leads to utilization of non-oxidative pathways for energy production and thus lactate accumulation in the brain, muscle and serum since pyruvate is not able to be shuttled into the TCA cycle. Severe lactic acidosis has been associated with thiamine deficiency [1] and worsened in these cases by glucose administrations. Although this may explain why thiamine deficiency is harmful, the association with causing Wernicke's Encephalopathy after glucose treatment remains unclear. But it seems some association likely exists.

In chronic alcoholics, thiamine absorption in the gut is inhibited for reasons that are not entirely clear. Subramanya and coworkers [2] recently described a mechanism of significant inhibition in carrier-mediated thiamine transport across the jejunal brush-border membrane associated with a significant reduction in levels of expression of thiamine transporter- 1 in rats that were chronically fed alcohol. This work is based on the previous discovery that thiamine absorption in human small intestinal brush-border membranes proceeds via a pH-dependent, electroneutral, carrier-mediated mechanism [3].

There are two reports [4] [5] citing acute encephalopathy in 5 profoundly malnourished patients after receiving IV glucose solutions. However, none of these patients developed symptoms after a single dose of glucose. Additionally these patients developed their symptoms over a course of days. Nevertheless, proponents of "thiamine before glucose" feel otherwise and conclude that if a blood glucose test reveals no hypoglycemia, thiamine should be administered

Table 1. Syndrome-hallmarks of disease.

\begin{tabular}{cc}
\hline Wernicke's Encephalopathy & $\begin{array}{c}\text { Mental status changes, ocular abnormalities, } \\
\text { gait ataxia }\end{array}$ \\
Wet Beriberi & High-output cardiac failure with edema \\
Dry Beriberi & High-output cardiac failure without edema \\
Neuritic Beriberi & Polyneuropathy \\
Gastrointestinal Beriberi & Abdominal pain, lactic acidosis, vomiting \\
\hline
\end{tabular}


before glucose if possible as part of therapy [6]. Patients in starvation states can still exhibit normal serum glucose levels however because degraded proteins are not replenished and serve as carbon sources for glucose synthesis.

Koguchi and colleagues [7] describe a case in which a 61 year-old man with a significant history of alcoholism presented with altered mental status and after starting IV glucose, the patient fell into a coma. No time frame is given in the report, but the patient already had radiographic evidence of Wernicke's Encephalopathy as evidenced on MRI imaging of the head during his hospitalization. As shown in Figure 1, MRI revealed hyperintense lesions of the mammillary bodies and horns of the fourth ventricles consistent with Wernicke's Encephalopathy. Although the patient did not receive thiamine until Day 5 of his admission and died 3 months later from pneumonia, the authors concluded that an infusion of glucose to patients with a known thiamine deficiency is potentially fatal.

There also exists several cases of Wernicke's Encephalopathy occurring in patients with hyperemesis gravidarum [8] [9], some indolently via IV glucose administration. For the most part, neurologic symptoms were already present before therapy. In these patients, body stores of thiamine can be depleted in weeks and chronically ill patients may already have critically low thiamine levels; RBC transketolase activity requires thiamine as a cofactor and an assay of enzymatic activity will reflect the robustness of thiamine stores. However in animal models, glucose loading had no significant clinical consequence when administered to rats fed a thiamine-deficient diet for 21 days. Advanced neurologic dysfunction with glucose administration was only seen in rats fed thiamine-deficient diets for 28 - 35 days and that ataxia and other neurologic sequela were clinically noted prior to glucose challenge [10] [Table 2].

\section{Conclusions and Summary of Recommendations}

Much controversy still exists regarding acute precipitation of Wernicke's Encephalopathy by administering glucose before thiamine in severely malnourished and alcoholic patients. The bottom line is that physiologically, this can happen but in most documented case reports, patients already had the manifestations of encephalopathy before they presented to the emergency department or other
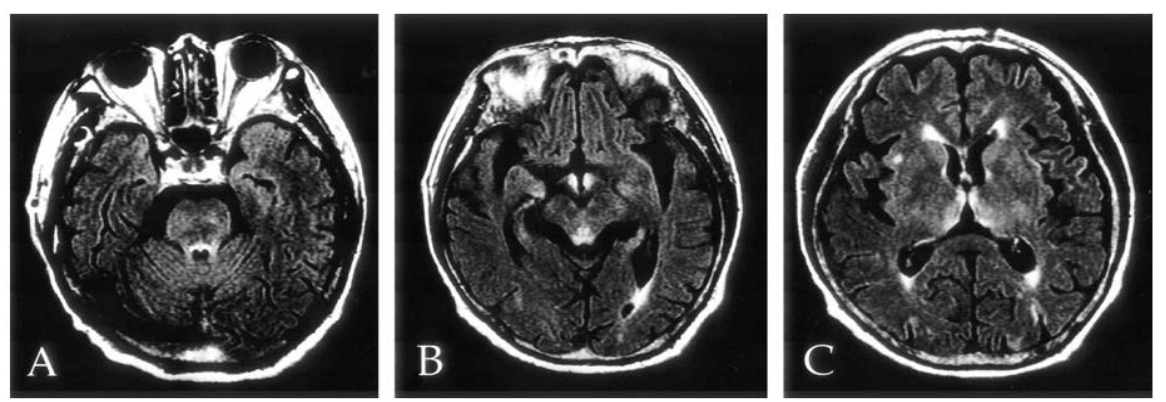

Figure 1. MRI image showing hyperintense lesions of the mammillary bodies and horns of the fourth ventricles consistent with Wernicke's Encephalopathy [7]. 
Table 2. Some causes of Wernicke's Encephalopathy.

- Alcohol Abuse

- AIDS

- Malignancy

- Hyperemesis gravidarum

- Prolonged total parenteral nutrition

- Post surgical patients

- Iatrogenic glucose loading in any predisposed patient

acute care seetting.

Moreover, the process has been shown to actually evolve throughout a hospital course, rather than the acute care and initial treatment in the emergency department. Even in one case example where acute decompensation is described, no actual time course is given, and thiamine administration was delayed until 5 days after glucose. The established recommendations are straightforward: do not delay glucose administration while waiting for thiamine. Furthermore, thiamine is best administered parentally, preferably IV versus IM, since GI absorption in malnourished patients is very low. The recommended dose is $250-500 \mathrm{mg}$ although there is little to no evidence-based studies supporting any dose (even doses as low as $2 \mathrm{mg}$ have been effective).

In summary, Wernicke's Encephalopathy can be hastened by glucose administration but not in the time frame of a typical single emergent care visit. Moreover, glucose administration should not be delayed while waiting for thiamine administration. We did not uproot any studies reporting immediate findings of Wernicke's Encephalopathy, coma or death from appropriately treating a malnourished patient. In these patients, glucose and thiamine, amongst other nutrients, should be replaced via IV administration as soon as possible, but treatment with glucose should not be delayed if awaiting thiamine.

\section{Conflicts of Interest}

The authors have no conflicts of interest to report.

\section{References}

[1] Klein, M., Weksler, N. and Gurman, G.M. (2004) Fatal Metabolic Acidosis Caused by Thiamine Deficiency. Journal of Emergency Medicine, 26, 301-303. https://doi.org/10.1016/j.jemermed.2003.11.014

[2] Subramanya, S.B., Subramanian, V.S. and Said, H.M. (2010) Chronic Alcohol Consumption and Intestinal Thiamin Absorption: Effects on Physiological and Molecular Parameters of the Uptake Process. American Journal of Physiology-Gastrointestinal and Liver Physiology, 299, G23-G31. https://doi.org/10.1152/ajpgi.00132.2010

[3] Dudeja, P.K., Tyagi, S., Kavilaveettil, R.J., Gill, R. and Said, H.M. (2011) Mechanism of Thiamine Uptake by Human Jejunal Brush-Border Membrane Vesicles. American Journal of Physiology: Cell Physiology, 281, C786-C792. https://doi.org/10.1152/ajpcell.2001.281.3.C786 
[4] Watson, A.J., Walker, J.F., Tomkin, G.H., Finn, M.M. and Keogh, J.A. (1981) Acute Wernickes Encephalopathy Precipitated by Glucose Loading. Irish Journal of Medical Science, 150, 301-303. https://doi.org/10.1007/BF02938260

[5] Drenick, E.J., Joven, C.B. and Swendseid, M.E. (1966) Occurrence of Acute Wernicke's Encephalopathy during Prolonged Starvation for the Treatment for Obesity. The New England Journal of Medicine, 274, 937-939.

https://doi.org/10.1056/NEJM196604282741705

[6] Hack, J.B. and Hoffman, R.S. (1998) Thiamine before Glucose to Prevent Wernicke Encephalopathy: Examining the Conventional Wisdom. JAMA, 279, 583-584. https://doi.org/10.1001/jama.279.8.583a

[7] Koguchi, K., Nakatsuji, Y., Abe, K. and Sakoda, S. (2004) Wernicke's Encephalopathy after Glucose Infusion. Neurology, 62, 512. https://doi.org/10.1212/01.WNL.0000099189.56741.A7

[8] Giugale, L.E., Young, O.M. and Streitman, D.C. (2015) Iatrogenic Wernicke Encephalopathy in a Patient with Severe Hyperemesis Gravidarum. Obstetrics \& Gynecology, 125, 1150-1152. https://doi.org/10.1097/AOG.0000000000000557

[9] Ohkoshi, N., Ishii, A. and Shoji, S. (1994) Wernicke's Encephalopathy Induced by Hyperemesis Gravidarum, Associated with Bilateral Caudate Lesions on Computed Tomography and Magnetic Resonance Imaging. European Neurology, 34, 177-180. https://doi.org/10.1159/000117034

[10] Zimitat, C. and Nixon, P.F. (1999) Glucose Loading Precipitates Acute Encephalopathy in Thiamin-Deficient Rats. Metabolic Brain Disease, 14, 1-20.

https://doi.org/10.1023/A:1020653312697 